\title{
Association of CETP Gene Variants with Atherogenic Dyslipidemia Among Thai Patients Treated with Statin
}

This article was published in the following Dove Press journal: Pharmacogenomics and Personalized Medicine

\author{
Pornpen Srisawasdi $\mathbb{D}^{\prime}$ \\ Punyanuch Rodcharoen' \\ Somlak Vanavanan (1D' \\ Anchalee Chittamma' \\ Chonlaphat Sukasem ${ }^{2}$ \\ Chalitpon $\mathrm{Na}$ nakorn ${ }^{2}$ \\ Charungthai Dejthevaporn (ID $^{3}$ \\ Martin $\mathrm{H} \mathrm{Kroll}^{4}$ \\ 'Division of Clinical Chemistry, \\ Department of Pathology, Faculty of \\ Medicine, Ramathibodi Hospital, Mahidol \\ University, Bangkok, Thailand; ${ }^{2}$ Division \\ of Pharmacogenomics and Personalized \\ Medicine, Department of Pathology, \\ Faculty of Medicine, Ramathibodi \\ Hospital, Mahidol University, Bangkok, \\ Thailand; ${ }^{3}$ Department of Medicine, \\ Faculty of Medicine, Ramathibodi \\ Hospital, Mahidol University, Bangkok, \\ Thailand; ${ }^{4}$ Quest Diagnostics, Secaucus, \\ NJ 07094, United States of America
}

Objective: Patients treated with statins for dyslipidemia may still have a residual risk of atherosclerotic cardiovascular disease (ASCVD). To determine whether genetic variants in the cholesteryl ester transport protein (CETP), rs3764261 (C>A), rs708272 (G>A), and rs12149545 (G>A) affect ASCVD risk, we studied the association of these variants with dyslipidemia in statin-treated patients.

Patients and Methods: We included 299 adult Thai patients treated with a statin (95 men and 204 women). Genotyping was performed by conducting a TaqMan real-time polymerase chain reaction-based analysis. We used logistic regression models adjusted for potential confounders of age, body mass index, blood pressure, insulin resistance, and statin dosage to analyze the association between CETP variants and atherogenic lipoprotein patterns.

Results: CETP polymorphisms of rs3764261 and rs708272, but not rs12149545, were significantly associated with high-density lipoprotein cholesterol (HDL-C), apoA-I, triglycerides, very low-density lipoprotein (VLDL)-C, and large LDL (LDL1-C) levels as well as mean LDL particle size (all $p<0.020$ ). However, no significant difference was observed in total cholesterol, LDL-C, or apoB levels by CETP variants. Regardless of sex, the combination of rs3764261 (CC genotype) and rs708272 (GG or GA genotypes) showed a stronger association with atherogenic dyslipidemia, including features of decreased HDL-C, elevated triglycerides, and LDL subclass pattern $\mathrm{B}$ (odds ratio $[\mathrm{OR}]=2.99,95 \%$ confidence interval [CI]: 1.78-5.02) compared with the single variant $\mathrm{rs} 3764261(\mathrm{OR}=2.11,95 \% \mathrm{CI}: 1.27-3.50)$ or rs708272 (OR $=2.12,95 \% \mathrm{CI}: 1.29-3.49)$.

Conclusion: The polymorphisms of CETP rs3764261 (CC genotype) and rs708272 (GG and GA genotypes) may have a higher susceptibility to atherogenic dyslipidemia. Testing for CETP rs3764261 and rs708272 may serve as a surrogate marker for lipid management in statin-treated patients, which may help individualize treatment for reducing the residual risk of ASCVD.

Keywords: residual atherosclerotic risk, statin treatment, cholesteryl ester transport protein, genetic polymorphisms, genetic risk

\section{Introduction}

Statin therapy is the first-line treatment for the primary prevention of atherosclerotic cardiovascular disease (ASCVD) in patients with major risk factors such as an elevated low-density lipoprotein (LDL) cholesterol (LDL-C) level, diabetes mellitus, metabolic syndrome, chronic kidney disease, and chronic inflammatory conditions. ${ }^{1}$ Statins inhibit 3-hydroxy-3-methylglutaryl-coenzyme A (HMG-CoA)
Correspondence: Pornpen Srisawasdi Division of Clinical Chemistry,

Department of Pathology, Faculty of

Medicine, Ramathibodi Hospital, Rama VI

Road, Ratchathewi, Bangkok 10400,

Thailand

Tel +66-2-20I-0008

Fax +66-2-354-7266

Email srisawasdip@yahoo.com
Pharmacogenomics and Personalized Medicine 202I:14 I-13

DovePress $\boldsymbol{f}$ in $\boldsymbol{\nabla}$ 
reductase, thus reducing hepatic cholesterol biosynthesis and leading to the upregulation of cell-surface LDL receptors, which act as lipid attachments for intracellular signaling molecules. ${ }^{2}$ Many randomized clinical trials have concluded that statins can prevent ASCVD. ${ }^{3-6}$ Still, the response to statins varies widely from patient to patient: the decrease in LDL-C levels after statin administration can range from $5 \%$ to $70 \% .^{7}$ A large population-based study, reflecting routine practice, demonstrated that $50 \%$ of patients on statin therapy did not exhibit the intended therapeutic benefit. ${ }^{8}$ Moreover, some clinical trials have indicated that even though statins effectively lower the LDL-C level in patients with type 2 diabetes, metabolic syndrome, and obesity, they continued to have a substantial residual risk of ASCVD. ${ }^{9,10}$ The residual risk can be contributed by hypertriglyceridemia, a low high-density lipoprotein cholesterol (HDL-C) level, and an increased number of small dense LDL (sdLDL) particles. $^{11,12}$

Atherogenic dyslipidemia, characterized by hypertriglyceridemia, a high number of sdLDL particles, and a low HDL-C level, occurs commonly in the insulinresistant state, which is a vital contributor to both macrovascular and microvascular residual risk. ${ }^{11-13}$ Cholesteryl ester transfer protein (CETP) affects the pathogenesis of atherogenic dyslipidemia. ${ }^{14,15}$ CETP is a hydrophobic protein mainly bound to HDL in plasma, which catalyzes the equimolar transfer of triglycerides and cholesterol esters between plasma HDL and triglyceride-rich lipoprotein particles [very lowdensity lipoprotein (VLDL) and LDL]. In individuals with hypertriglyceridemia, elevated CETP activity acts on the increased number of VLDL particles, preferentially transferring triglycerides to $\mathrm{HDL}$ and $\mathrm{LDL}$ particles. $^{12,13}$ The redistribution of these lipids enhances CETP activity, leading to size and composition changes in LDL and HDL particles, thereby reducing the HDL-C level and increasing the atherogenicity of sdLDL particles.

Statins and CETP both affect the plasma lipid concentration and composition, and CETP activity decreases during statin treatment. ${ }^{15-17}$ The interaction between CETP and response to statin therapy may be relevant to mixed dyslipidemia. ${ }^{18,19}$ CETP activity has a strong genetic component, and CETP gene variants can contribute to ASCVD risk. ${ }^{20-23}$ Numerous studies have investigated the association between CETP polymorphisms and metabolic abnormalities such as obesity, hypertension, and insulin resistance. ${ }^{24-26}$ CETP rs3764261 and rs708272 (also called Taq1B) variants have been associated with decreased plasma CETP activity, resulting in a higher HDL-C level and a lower atherogenicity index in different ethnic populations. ${ }^{18,21-23,27-29}$ Recently, two Chinese studies have demonstrated that the variants rs1800775, rs 12149545 , and rs 711752 were closely linked to dyslipidemia and metabolic syndrome. ${ }^{30,31}$ However, the findings of rs1800775 and rs711752 variants have been less conclusive. ${ }^{32,33}$

In this study, we determined whether the genetic variants of CETP—rs3764261, rs708272, and rs12149545-are associated with dyslipidemia, particularly the atherogenic lipoprotein phenotype including a decreased HDL-C level, an elevated triglyceride level, and a decreased mean LDL particle size, and therefore with the residual CVD risk in Thai patients treated with statins. Even though such associations have been observed for other populations, this is the first study in the Thai population. Furthermore, because there are sex differences in the plasma lipid and lipoprotein metabolism, ${ }^{34}$ we conducted the study to determine whether associations between the CETP variants with atherogenic dyslipidemia had sex-specific characteristics. Identifying CETP polymorphisms relevant to atherogenic dyslipidemia in statin-treated patients can aid in more precise interventions to treat residual cardiovascular risk.

\section{Patients and Methods}

\section{Study Patients}

This cross-sectional study included 306 adult patients who received statin monotherapy for $\geq 6$ months at Ramathibodi Hospital (Bangkok, Thailand). Patients with a known history of severe medical conditions such as cancer, end-stage renal disease, hepatobiliary diseases, severe acute illness, and thyroid disorders were excluded. Patients provided informed consent, and their blood was collected once for genotyping and biochemical analyses. Data related to patient demographics, statin treatment (type and dose), and other concomitant medications were collected from medical and pharmacy records. Height, weight, and body mass index (BMI) data were collected at the time of enrollment.

The study protocol was approved by the ethics committee of the Faculty of Medicine, Ramathibodi Hospital, Mahidol University (MURA2019/984). All patients provided written informed consent. 


\section{Single-Nucleotide Variant Selection and Genotyping Analysis}

Candidate CETP single-nucleotide variant, SNV (formerly SNP) were selected based on the significant association with HDL-C and CETP activity in studies on CETP variants. ${ }^{27-31}$ In addition, the minor allele frequency of the SNV found in Han Chinese ethnicity in Beijing according to the International HapMap Project database (http://hapmap.ncbi.nlm.nih.gov) was $>10 \%$. In total, three CETP variants were selected: CETP rs708272, rs3764261, and rs 12149545 .

Genomic DNA was extracted from EDTA blood by using the MagNA Pure automated extraction system (Roche Applied Science, Penzberg, Germany). The protocol for DNA extraction has been described in our previous study. ${ }^{35}$ Genotyping of the CETP polymorphisms was performed by conducting a TaqMan SNP genotyping assay on an Applied Biosystems ${ }^{\circledR}$ ViiA $^{\mathrm{TM} 7}$ Real-Time polymerase chain reaction (PCR) System (ABI, Foster City, CA, USA). The extracted DNA was amplified through PCR by using the primers of CETP c.2490C $>$ A, rs3764261; c.5454G $>A$, rs708272; and c.2327G $>A$, rs12149545. The genotyping analysis was performed according to manufacturer's recommendations.

\section{Biochemical Measurements}

Serum total cholesterol (TC), triglycerides, LDL-C, HDL-C, apolipoprotein A-I (apoA-I), and apolipoprotein B (apoB) concentrations, as well as plasma glucose levels, were measured using the Siemens Chemistry System (Siemens Healthcare Diagnostics, Tarrytown, NY, USA). Our laboratory has been certified by the Centers for Disease Control and Prevention-Lipid Standardization Program. Consequently, the analytical performances of TC, triglycerides, HDL-C, apoA-I, and apoB measurements were within the acceptable criteria of the National Cholesterol Education Program.

Lipoprotein subclass analysis was performed using a linear polyacrylamide tube gel electrophoresis method (Quantimetrix Lipoprint ${ }^{\mathrm{TM}}$, Redondo Beach, CA, USA). ${ }^{36}$ The cholesterol level in each subfraction-VLDL; MidC, MidB, and MidA (intermediate low-density lipoprotein, IDL); LDL1 and LDL2 (large-buoyant LDL), LDL3 to LDL7 (sdLDL), and HDL-was measured. Mean LDL particle sizes were computed. Patients with predominantly large-buoyant LDL (mean LDL particle sizes $>26.5 \mathrm{~nm}$ ) were classified as LDL subclass pattern A, and those with predominantly sdLDL (mean LDL particle sizes $\leq 26.5$ nm) were classified as pattern B.

Hemoglobin A1c (HbAlc) and insulin levels were quantified using the immunoassay on Cobas Integra (Roche Diagnostics) and Immulite (Siemens Healthcare Diagnostics), respectively. To estimate the degree of insulin resistance in an individual patient, we used the homeostasis model assessment of insulin resistance (HOMA-IR) score, which was calculated as follows: HOMA-IR = fasting insulin $(\mu \mathrm{U} / \mathrm{mL}) \times$ fasting glucose $(\mathrm{mmol} / \mathrm{L}) / 22.5{ }^{37}$

\section{Statistical Analyses}

Continuous data are expressed as medians (interquartile range) or means (standard deviation), and categorical variables are reported as numbers and percentages. Differences among the groups were assessed using the $\chi^{2}$ test, Kruskal-Wallis test, or $t$-test as appropriate. The recessive genetic model was used for CETP rs3764261 (CC vs $\mathrm{AA}+\mathrm{CA}$ ) and CETP rs12149545 (GG vs AA $+\mathrm{GA}$ ), and the dominant genetic model was used for CETP rs708272 (GG+GA vs AA). The association of CETP polymorphisms with dyslipidemia was assessed by calculating odds ratios (ORs) and corresponding 95\% confidence intervals (CIs). A backward stepwise multivariable logistic regression analysis was used to assess whether one or more genetic factors predict the atherogenic dyslipidemia complex, including all features of the decreased HDLC level, elevated triglyceride level, and LDL subclass pattern B, after adjustment for sex, age, BMI, systolic and diastolic blood pressure, HOMA-IR score, and statin dosage. Outcomes were considered statistically significant when $p<0.05$, and all analyses were performed using SPSS, version 18.0 (SPSS Inc., Chicago, IL, USA).

The Hardy-Weinberg equilibrium and linkage disequilibrium coefficients were analyzed using Haploview program v4.2 (Broad Institute, Cambridge, MA, USA).

\section{Results}

\section{Characteristics and Biomarkers of the Study Population}

We included 299 patients (95 men and 204 women; age 37-92 years); seven patients were excluded because of a highly elevated creatinine $(n=5)$ level or chylomicrons or highly elevated triglycerides $(n=2)$. Table 1 summarizes participants' demographic and biochemical test results stratified by sex. The mean (standard deviation) age was 63.0 (8.3) years. Statins prescribed were 
Table I Clinical Parameters and Biomarker Levels ${ }^{\dagger}$

\begin{tabular}{|c|c|c|c|c|}
\hline & All $(n=299)$ & Men $(n=95)$ & Women $(n=204)$ & p-value \\
\hline \multicolumn{5}{|c|}{ Clinical parameter, mean (range) } \\
\hline Age, years & $63.0(37-92)$ & $62.7(38-92)$ & $63.3(37-89)$ & 0.707 \\
\hline Systolic BP (mm Hg) & $132.3(9|-2| 0)$ & $131.7(92-180)$ & $132.6(9|-2| 0)$ & 0.860 \\
\hline Diastolic BP (mm Hg) & $76.0(44-120)$ & $76.2(44-100)$ & $76.0(50-120)$ & 0.852 \\
\hline Body mass index, $\mathrm{kg} / \mathrm{m}^{2}$ & $25.9(15.7-49.5)$ & $25.9(15.7-49.5)$ & $26.0(17.4-42.3)$ & 0.842 \\
\hline \multicolumn{5}{|l|}{ Statin dosage } \\
\hline Low intensity & $176(58.9)$ & $50(52.6)$ & $126(61.8)$ & 0.306 \\
\hline Moderate intensity & $116(38.8)$ & $42(44.2)$ & $74(36.3)$ & \\
\hline High intensity & $7(2.3)$ & $3(3.2)$ & $4(2.0)$ & \\
\hline \multicolumn{5}{|l|}{ CETP Polymorphisms } \\
\hline \multicolumn{5}{|l|}{ rs376426I (C>A) genotype } \\
\hline $\mathrm{CC}$ & $126(42.1)$ & $40(42.1)$ & $86(42.2)$ & 0.822 \\
\hline $\mathrm{CA}$ & I $40(46.8)$ & $43(45.3)$ & $97(47.5)$ & \\
\hline AA & $33(11.0)$ & $12(12.6)$ & $21(10.3)$ & \\
\hline \multicolumn{5}{|l|}{ rs708272 (G>A) genotype } \\
\hline GG & I0I (33.8) & $34(35.8)$ & $67(32.8)$ & 0.760 \\
\hline GA & $153(5 \mid .2)$ & $49(51.6)$ & $104(5 \mid .0)$ & \\
\hline AA & $45(15.0)$ & $12(12.6)$ & $33(16.2)$ & \\
\hline \multicolumn{5}{|c|}{ rs I2 I49545 (G>A) genotype } \\
\hline GG & $193(64.5)$ & $61(64.2)$ & $132(64.7)$ & 0.993 \\
\hline GA & $97(32.5)$ & $31(32.6)$ & $66(32.4)$ & \\
\hline AA & $9(3.0)$ & $3(3.2)$ & $6(2.9)$ & \\
\hline \multicolumn{5}{|l|}{ Biomarkers } \\
\hline Fasting glucose, $\mathrm{mmol} / \mathrm{L}$ & $5.78(5.17-7.15)$ & $6.44(5.33-8.14)$ & $5.67(5.12-6.82)$ & 0.001 \\
\hline Insulin, $\mu \mathrm{lU} / \mathrm{mL}$ & $7.0(4.8-10.7)$ & $7.3(4.8-10.8)$ & $7.0(4.7-10.7)$ & 0.965 \\
\hline HOMA-IR & $1.98(1.19-3.17)$ & $2.05(1.37-3.72)$ & $1.90(1.14-3.04)$ & 0.157 \\
\hline $\mathrm{HbAlc}(\mathrm{mmol} / \mathrm{mol})$ & $47.7(4 \mid .2-58.7)$ & 5I.I (4I.6-62.I) & $46.7(40.1-56.5)$ & 0.039 \\
\hline
\end{tabular}

Notes: ${ }^{\dagger}$ Continuous data are given as median (interquartile range). Categorical variables are presented as numbers (\%). All biochemical measures are given in Système International units; conversions to conventional units are as follows: fasting glucose $(\mathrm{mg} / \mathrm{dL})$, multiply by $18.02 ; \mathrm{HbAlc}(\%)$, use the formula: [0.09I5 $\mathrm{HbAlc}(\mathrm{mmol} / \mathrm{mol})+2.15]$.

Abbreviations: BP, blood pressure; HOMAIR, homeostasis model assessment of insulin resistance.

simvastatin, atorvastatin, pitavastatin, and rosuvastatin. Most patients were treated with low-intensity statins, followed by moderate- and high-intensity statins; no sex differences were observed with respect to the statin intensity. The genotype distributions of CETP rs3764261, rs708272, and rs12149545 polymorphisms did not differ significantly between sex. The Hardy-Weinberg equilibrium probability values were $0.458,0.422$, and 0.593 , respectively. The linkage disequilibrium structure between the CETP polymorphisms is shown in Figure 1.

\section{Effects of CETP Polymorphisms on Lipid Biomarker Levels}

The plasma lipid, lipoprotein, and apolipoprotein levels (Table 2) as well as the lipoprotein subclass and mean LDL particle size (Table 3) were compared among the
CETP genotype groups. The median concentrations of HDL-C, triglycerides, and mean apoA-I differed significantly (all $p \leq 0.010$ ) among participants with different CETP polymorphisms of rs3764261 and rs708272. Participants with common homozygous rs3764261 CC genotype had significantly lower median HDL-C and mean apoA-I levels (17.8\% and $8.2 \%$, respectively) and a higher median triglycerides level (41.5\%) than did those with the minor AA genotype. Similarly, participants with GG genotype for rs708272 had significantly lower median HDL-C and mean apoA-I levels (12.1\% and 8.3\%, respectively) and a higher median triglycerides level (35.0\%) than did those with the minor AA genotype. No significant differences in TC, LDL-C, and apoB levels between genetic polymorphisms were observed. No significant differences in lipid biomarker levels were seen among the rs12149545 polymorphism groups. 


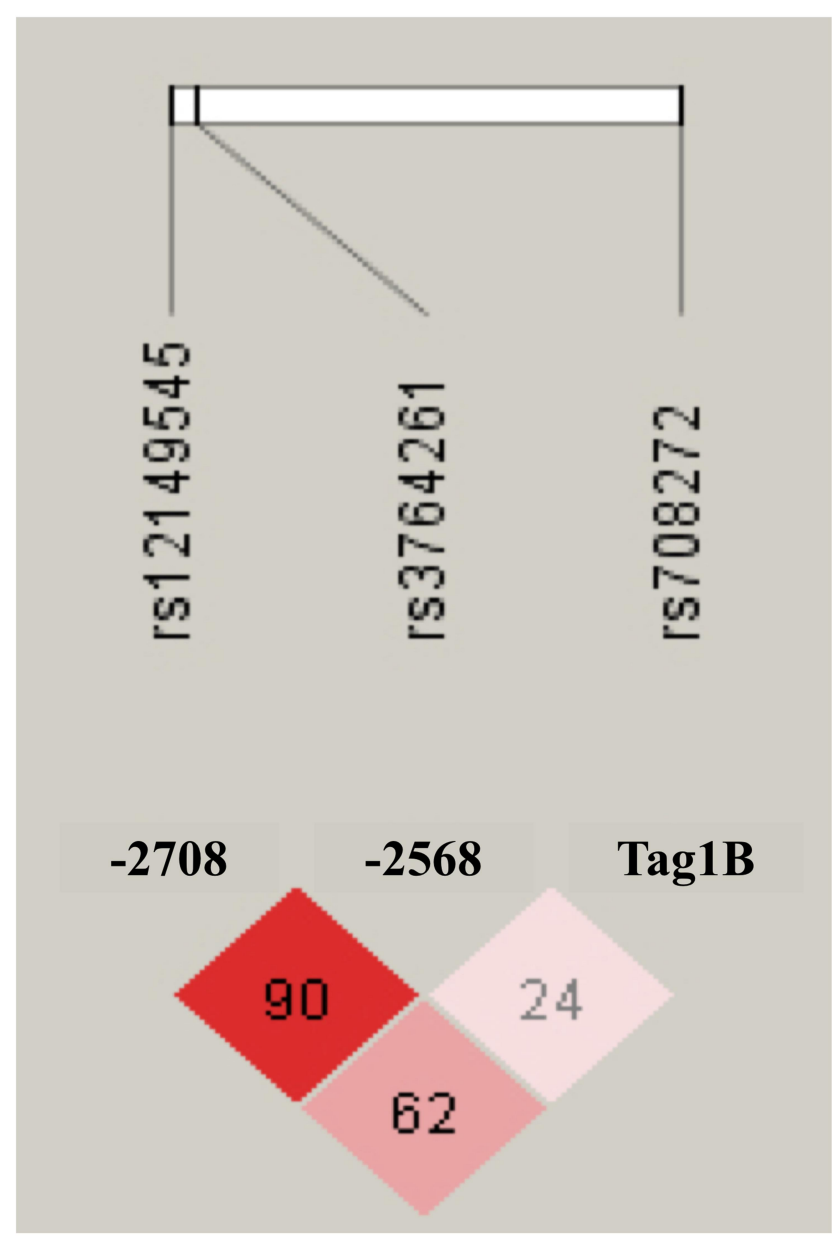

Figure I Linkage disequilibrium pattern between CETP polymorphisms of rs376426I, rs708272 and rs I2149545. The numbers in each box correspond to measured linkage disequilibrium coefficients among all SNV $(n=299)$.

Concerning LDL subclasses, the rs3764261 CC genotype and rs708272 GG genotype showed higher VLDL-C levels, lower LDL1-C levels, and lower mean LDL particle size than did AA genotype carriers. No significant differences were observed among the rs12149545 polymorphism groups.

\section{Associations of CETP Polymorphisms with Dyslipidemia}

Table 4 presents CETP genotype distributions in patients with normal and abnormal plasma lipid, lipoprotein, and LDL subclass patterns and those treated with statin stratified by sex. The rs3764261 and rs708272 polymorphisms were significantly associated with each feature of atherogenic dyslipidemia, including decreased HDL-C levels, elevated triglyceride levels, VLDL-C levels, and LDL subclass pattern B. Patients with the CC rs3764261 genotype had the highest risk of hypoHDL-cholesterolemia (42.1\%), hypertriglyceridemia (57.9\%),
hyper-VLDL-cholesterolemia (66.7\%), and LDL subclass pattern B (49.2\%), followed by those with the CA genotype $(26.4 \%, 37.9 \%, 39.3 \%$, and $31.4 \%$, respectively) and the AA genotype $(9.1 \%, 36.4 \%, 33.3 \%$, and $15.2 \%$, respectively). Using the recessive genetic model ( $\mathrm{CC}$ vs $\mathrm{AA}+\mathrm{CA}$ ), the ORs (95\% CIs) were 2.414 (1.464-3.980), 2.289 (1.432-3.657), 3.242 (2.004-5.245), and 2.452 (1.515-3.966) for reduced HDL-C levels, elevated triglycerides, elevated VLDL-C levels, and LDL subclass pattern B, respectively. Sexstratified analysis revealed significant associations in both sexes. According to the rs 708272 polymorphism, similar associations were noted. The GG homozygous had the highest risk of hypo-HDL-cholesterolemia (35.6\%), hypertriglyceridemia (51.5\%), hyper-VLDL-cholesterolemia (62.4\%), and LDL subclass pattern B $(45.5 \%)$, followed by those with the GA genotype $(34.6 \%, 51.6 \%, 53.6 \%$, and $38.6 \%$, respectively) and the AA genotype $(8.9 \%, 15.6 \%, 11.1 \%$, and $13.3 \%$, respectively). Using the dominant genetic model (GG+GA vs AA), the ORs $(95 \%$ CI) were 5.529 (1.918-15.935), 5.782 (2.489-13.431), 10.642 (4.065-27.859), and 4.581 (1.872-11.211) for decreased HDL-C levels, elevated triglycerides, elevated VLDL-C levels, and LDL subclass pattern B, respectively. Sex-stratified analysis revealed significant associations in both sexes. For polymorphism rs 12149545, using the recessive genetic model (GG vs AA+GA), the ORs did not demonstrate a significant association with dyslipidemia in either sex, except for elevated VLDL-C levels in men (OR, 2.487; 95\% CI, 1.048-5.903).

To assess the association between CETP polymorphisms and the atherogenic dyslipidemia complex (all features of reduced HDL-C, elevated triglycerides, and atherogenic lipoprotein pattern B), we evaluated their contributions by performing a binary logistic regression (Table 5). In Model 1, all CETP genetic variants and the clinical factors of sex, age, BMI, systolic and diastolic blood pressure, HOMA-IR, and statin dosage were considered independent variables. Furthermore, subgroup analyses based on sex were conducted. Overall, both CETP rs3764261 and rs708272 exhibited significant associations with atherogenic dyslipidemia after adjustment for confounders (OR, 2.11; 95\% CI, 1.27-3.50 and OR, 2.12; 95\% CI, 1.29-3.49, respectively). When stratified by sex, both rs3764261 and rs708272 exhibited significant associations for women (OR, 2.54; 95\% CI, 1.26-5.11 and OR, 2.37; 95\% CI, 1.24-4.53, respectively) but not for men. To evaluate the potential gene-gene interaction effect, we combined the polymorphisms of rs3764261 (CC genotype/A allele) with rs708272 (G allele/AA genotype) by 
Table 2 Plasma Lipid, Lipoprotein and Apolipoprotein Biomarkers Among CETP Variants in Patients Treated with Statin

\begin{tabular}{|c|c|c|c|c|c|c|}
\hline & \multicolumn{6}{|l|}{ Lipid Biomarkers $^{\dagger}$} \\
\hline & $\begin{array}{l}\text { Total cholesterol } \\
(\mathrm{mmol} / \mathrm{L})\end{array}$ & $\begin{array}{l}\text { LDL-C } \\
(\mathrm{mmol} / \mathrm{L})\end{array}$ & $\begin{array}{l}\text { HDL-C } \\
(\mathrm{mmol} / \mathrm{L})\end{array}$ & $\begin{array}{l}\text { Triglycerides } \\
\text { (mmol/L) }\end{array}$ & $\begin{array}{l}\text { Apolipoprotein-Al } \\
\text { (mg/dL) }\end{array}$ & $\begin{array}{l}\text { Apolipoprotein-B } \\
\text { (mg/dL) }\end{array}$ \\
\hline \multicolumn{7}{|c|}{ CETP rs376426I (C>A) } \\
\hline$C C(n=126)$ & $4.87(4.37-5.34)$ & $2.71(0.74)$ & $1.26(1.06-1.55)$ & $1.98(1.22-2.44)$ & $157.6(24.2)$ & $90.1(79.3-106.0)$ \\
\hline$C A(n=140)$ & $4.90(4.45-5.31)$ & $2.74(0.87)$ & $1.40(1.19-1.70)$ & $1.52(1.11-2.03)$ & $164.3(27.2)$ & $86.7(76.5-102.0)$ \\
\hline $\mathrm{AA}(\mathrm{n}=33)$ & $5.10(4.75-5.53)$ & $3.02(0.61)$ & $1.53(1.39-1.88)$ & $1.40(1.13-1.78)$ & $171.7(25.6)$ & $86.0(77.6-99.5)$ \\
\hline$p$-value & 0.180 & 0.118 & $<0.001$ & 0.004 & 0.010 & 0.597 \\
\hline \multicolumn{7}{|c|}{ CETP rs708272 (G>A) } \\
\hline GG $(n=|0|)$ & $4.84(4.39-5.31)$ & $2.72(0.78)$ & $1.32(1.11-1.57)$ & $1.79(1.18-2.27)$ & $158.6(25.7)$ & 86.4 (78.2-99.8) \\
\hline GA $(n=153)$ & $4.95(4.43-5.54)$ & $2.77(0.83)$ & $1.35(1.10-1.66)$ & $1.73(1.16-2.44)$ & $161.6(26.6)$ & $88.6(78.0-108.5)$ \\
\hline $\mathrm{AA}(\mathrm{n}=45)$ & $4.92(4.5 \mid-5.2 I)$ & $2.79(0.72)$ & $1.50(1.30-1.77)$ & $1.32(1.05-1.53)$ & $173.0(23.2)$ & $87.7(76.5-94.6)$ \\
\hline p-value & 0.617 & 0.852 & 0.006 & 0.001 & 0.008 & 0.398 \\
\hline \multicolumn{7}{|c|}{ CETP rs $12149545(\mathrm{G}>\mathrm{A})$} \\
\hline$G G(n=193)$ & $4.95(4.43-5.37)$ & $2.77(0.77)$ & $1.35(1.14-1.61)$ & $1.66(1.16-2.28)$ & $161.7(25.6)$ & $88.9(78.8-101.0)$ \\
\hline $\mathrm{GA}(\mathrm{n}=97)$ & $4.84(4.42-5.34)$ & $2.74(0.86)$ & $1.37(1.17-1.72)$ & $1.58(1.13-2.27)$ & $162.3(27.0)$ & $87.1(75.9-107.0)$ \\
\hline $\mathrm{AA}(\mathrm{n}=9)$ & $4.95(4.60-5.44)$ & $2.69(0.65)$ & $1.71(1.27-1.98)$ & $1.37(1.09-1.85)$ & $175.3(27.2)$ & $84.2(75.1-98.9)$ \\
\hline p-value & 0.756 & 0.937 & 0.119 & 0.516 & 0.312 & 0.666 \\
\hline
\end{tabular}

Notes: ${ }^{\dagger}$ The parametric variables are given as mean (standard deviation) and the nonparametric variables are given as median (interquartile range). All biochemical measures are given in Système International units; conversions to conventional units are as follows: cholesterol (mg/dL), multiply by 38.6 ; triglycerides (mg/dL), multiply by 88.5 . Abbreviations: LDL-C, low-density lipoprotein cholesterol; HDL-C, high-density lipoprotein cholesterol.

Table 3 LDL Subclass Among CETP Variants in Patients Treated with Statin

\begin{tabular}{|c|c|c|c|c|c|c|}
\hline & \multicolumn{6}{|c|}{ LDL Subclass $(\mathrm{mmol} / \mathrm{L})^{\dagger}$} \\
\hline & VLDL-C & IDL-C & LDLI-C & LDL2-C & LDL3-C to LDL7-C & Mean LDL particle size $(\mathrm{nm})$ \\
\hline \multicolumn{7}{|c|}{ CETP rs376426I (C>A) } \\
\hline$C C(n=126)$ & $0.84(0.65-1.09)$ & $1.15(0.96-1.37)$ & $0.70(0.54-0.93)$ & $0.57(0.4 \mathrm{I}-0.73)$ & $0.23(0.08-0.52)$ & $26.52(26.05-26.99)$ \\
\hline$C A(n=140)$ & $0.70(0.65-0.85)$ & $1.22(1.04-1.40)$ & $0.80(0.62-1.01)$ & $0.57(0.4 \mathrm{I}-0.75)$ & $0.13(0.08-0.39)$ & $26.82(26.35-27.00)$ \\
\hline $\mathrm{AA}(\mathrm{n}=33)$ & $0.70(0.60-0.82)$ & $1.22(1.01-1.50)$ & $0.93(0.79-1.05)$ & $0.60(0.44-0.74)$ & $0.10(0.08-0.19)$ & $26.87(26.66-27.04)$ \\
\hline$p$-value & 0.002 & 0.008 & $<0.001$ & 0.910 & 0.112 & 0.017 \\
\hline \multicolumn{7}{|c|}{ CETP rs708272 (G>A) } \\
\hline$G G(n=\mid 01)$ & $0.80(0.67-1.01)$ & $1.19(0.96-1.37)$ & $0.72(0.54-0.91)$ & $0.54(0.4 \mathrm{I}-0.73)$ & $0.18(0.06-0.47)$ & $26.65(26.15-27.05)$ \\
\hline GA $(n=153)$ & $0.78(0.65-1.02)$ & $1.19(1.01-1.37)$ & $0.78(0.60-1.00)$ & $0.60(0.4 I-0.75)$ & $0.18(0.08-0.47)$ & $26.67(26.12-26.97)$ \\
\hline AA $(n=45)$ & $0.67(0.57-0.71)$ & $1.22(1.05-1.44)$ & $0.96(0.79-1.10)$ & $0.60(0.44-0.74)$ & $0.13(0.05-0.21)$ & $26.90(26.66-27.04)$ \\
\hline p-value & $<0.001$ & 0.576 & $<0.001$ & 0.708 & 0.039 & 0.016 \\
\hline \multicolumn{7}{|c|}{ CETP rs I 2 |49545 (G>A) } \\
\hline GG $(n=193)$ & $0.78(0.65-1.01)$ & $1.19(1.01-1.40)$ & $0.78(0.57-1.01)$ & $0.57(0.4 \mathrm{I}-0.75)$ & $0.16(0.08-0.45)$ & $26.68(26.17-27.00)$ \\
\hline $\mathrm{GA}(\mathrm{n}=97)$ & $0.73(0.62-0.91)$ & I.I7 (0.98-1.37) & $0.80(0.60-1.00)$ & $0.60(0.4 \mathrm{I}-0.76)$ & $0.16(0.08-0.44)$ & $26.72(26.34-27.00)$ \\
\hline $\mathrm{AA}(\mathrm{n}=9)$ & $0.67(0.54-0.89)$ & $1.30(1.06-1.52)$ & $0.96(0.84-1.02)$ & $0.47(0.40-0.75)$ & $0.08(0.05-0.23)$ & $26.96(26.5 \mathrm{I}-27.16)$ \\
\hline p-value & 0.097 & 0.534 & 0.206 & 0.799 & $0.44 I$ & 0.245 \\
\hline
\end{tabular}

Note: ${ }^{\dagger}$ Data are given as median (interquartile range).

Abbreviations: VLDL-C, very low-density lipoprotein cholesterol; IDL-C, intermediate density lipoprotein cholesterol; LDLI-C and LDL2-C, large-buoyant low-density lipoprotein cholesterol; LDL3-C to LDL7-C, small-dense low-density lipoprotein cholesterol.

generating three groups of rs3764261/rs708272 (Table 1S). The proportions of patients with various numbers of lipid abnormalities in each genotype of CETP rs3764261, rs708272 polymorphisms, and combined rs3764261/rs708272 are displayed in supplementary Figure 1S. The potential combination of rs3764261 and rs708272 was used in Model 2. The combination of rs3764261 (CC genotype) and rs708272 (GG or GA 
Table 4 Genotype Frequency of CETP Variants Among Patients without and with Dyslipidemia ${ }^{\dagger}$

\begin{tabular}{|c|c|c|c|c|c|c|}
\hline \multirow[b]{2}{*}{ HDL-cholesterol } & \multicolumn{2}{|l|}{ All, n (\%) } & \multicolumn{2}{|l|}{ Men, n (\%) } & \multicolumn{2}{|l|}{ Women, n (\%) } \\
\hline & $\begin{array}{l}M \geq 1.04 \mathrm{mmol} / \mathrm{L} \\
W \geq 1.30 \mathrm{mmol} / \mathrm{L} \\
(\mathrm{n}=206)\end{array}$ & $\begin{array}{l}M<1.04 \mathrm{mmol} / \mathrm{L} \\
W<1.30 \mathrm{mmol} / \mathrm{L} \\
(\mathrm{n}=93)\end{array}$ & $\begin{array}{l}\geq 1.04 \mathrm{mmol} / \mathrm{L} \\
(\mathrm{n}=7 \mathrm{I})\end{array}$ & $\begin{array}{l}<1.04 \mathrm{mmol} / \mathrm{L} \\
(\mathrm{n}=24)\end{array}$ & $\begin{array}{l}\geq 1.30 \mathrm{mmol} / \mathrm{L} \\
(\mathrm{n}=135)\end{array}$ & $\begin{array}{l}<1.30 \mathrm{mmol} / \mathrm{L} \\
(\mathrm{n}=69)\end{array}$ \\
\hline \multicolumn{7}{|l|}{ CETP rs376426I (C>A) } \\
\hline$C C, n=40(M) ; 86(W)$ & $73(57.9)$ & $53(42.1)$ & $25(62.5)$ & $15(37.5)$ & $48(55.8)$ & $38(44.2)$ \\
\hline$C A, n=43(M) ; 97(W)$ & $103(73.6)$ & $37(26.4)$ & $35(81.4)$ & $8(18.6)$ & $68(70.1)$ & $29(29.9)$ \\
\hline $\mathrm{AA}, \mathrm{n}=12(\mathrm{M}) ; 2 \mathrm{I}(\mathrm{W})$ & $30(90.9)$ & $3(9.1)$ & II (9I.7) & $\mathrm{I}(8.3)$ & $19(90.5)$ & $2(9.5)$ \\
\hline OR $(95 \% \mathrm{Cl})^{\ddagger}$ & \multicolumn{2}{|l|}{$2.414(1.464-3.980)$} & \multicolumn{2}{|c|}{$3.067(1.175-8.003)$} & \multicolumn{2}{|c|}{$2.222(1.230-4.012)$} \\
\hline p-value & \multicolumn{2}{|l|}{0.001} & \multicolumn{2}{|c|}{0.022} & \multicolumn{2}{|l|}{0.008} \\
\hline \multicolumn{7}{|l|}{ CETP rs708272 (G>A) } \\
\hline GG, $n=34(M) ; 67(W)$ & $65(64.4)$ & $36(35.6)$ & $25(73.5)$ & $9(26.5)$ & $40(59.7)$ & $27(40.3)$ \\
\hline $\mathrm{GA}, \mathrm{n}=49(\mathrm{M}) ; 104(\mathrm{~W})$ & $100(65.4)$ & $53(34.6)$ & $34(69.4)$ & $15(30.6)$ & $66(63.5)$ & $38(36.5)$ \\
\hline$A A, n=12(M) ; 33(W)$ & 41 (9I.I) & $4(8.9)$ & $12(100)$ & $0(0.0)$ & $29(87.9)$ & $4(12.1)$ \\
\hline OR $(95 \% \mathrm{Cl})^{\S}$ & \multicolumn{2}{|l|}{$5.529(1.918-15.935)$} & \multirow{2}{*}{\multicolumn{2}{|c|}{ - I }} & \multirow{2}{*}{\multicolumn{2}{|c|}{$\begin{array}{l}4.446(1.495-13.223) \\
0.007\end{array}$}} \\
\hline p-value & 0.002 & & & & & \\
\hline \multicolumn{7}{|l|}{ CETP rs I2I $49545(\mathrm{G}>\mathrm{A})$} \\
\hline $\mathrm{GG}, \mathrm{n}=61(\mathrm{M}) ; 132(\mathrm{~W})$ & $129(66.8)$ & $64(33.2)$ & $42(68.9)$ & $19(31.1)$ & $87(65.9)$ & $45(34.1)$ \\
\hline $\mathrm{GA}, \mathrm{n}=3 \mathrm{I}(\mathrm{M}) ; 66(\mathrm{~W})$ & $69(71.1)$ & $28(28.9)$ & $26(83.9)$ & $5(16.1)$ & $43(65.2)$ & $23(43.8)$ \\
\hline $\mathrm{AA}, \mathrm{n}=3(\mathrm{M}) ; 6(\mathrm{~W})$ & $8(88.9)$ & I (II.I) & $3(100.0)$ & $0(0.0)$ & $5(83.3)$ & I (16.7) \\
\hline OR $(95 \% \mathrm{Cl})^{\ddagger}$ & \multicolumn{2}{|l|}{$1.317(0.782-2.219)$} & \multicolumn{2}{|c|}{$2.624(0.880-7.826)$} & \multirow{2}{*}{\multicolumn{2}{|c|}{$\begin{array}{l}1.034(0.563-1.900) \\
0.913\end{array}$}} \\
\hline$p$-value & 0.301 & & 0.084 & & & \\
\hline Triglycerides & $\begin{array}{l}<1.70 \mathrm{mmol} / \mathrm{L} \\
(\mathrm{n}=161)\end{array}$ & $\begin{array}{l}\geq 1.70 \mathrm{mmol} / \mathrm{L} \\
(\mathrm{n}=138)\end{array}$ & $\begin{array}{l}<1.70 \mathrm{mmol} / \mathrm{L} \\
(\mathrm{n}=43)\end{array}$ & $\begin{array}{l}\geq 1.70 \mathrm{mmol} / \mathrm{L} \\
(\mathrm{n}=52)\end{array}$ & $\begin{array}{l}<1.70 \mathrm{mmol} / \mathrm{L} \\
(\mathrm{n}=118)\end{array}$ & $\begin{array}{l}\geq 1.70 \mathrm{mmol} / \\
L \\
(\mathrm{n}=86)\end{array}$ \\
\hline \multicolumn{7}{|l|}{ CETP rs376426I (C>A) } \\
\hline$C C, n=40(M) ; 86(W)$ & $53(42.1)$ & $73(57.9)$ & $13(32.5)$ & $27(67.5)$ & $40(46.5)$ & $46(53.5)$ \\
\hline$C A, n=43(M) ; 97(W)$ & $87(62.1)$ & $53(37.9)$ & $24(55.8)$ & $19(44.2)$ & $63(64.9)$ & $34(35.1)$ \\
\hline $\mathrm{AA}, \mathrm{n}=12(\mathrm{M}) ; 21(\mathrm{~W})$ & $21(63.6)$ & $12(36.4)$ & $6(50.0)$ & $6(50.0)$ & $15(7 \mid .4)$ & $6(28.6)$ \\
\hline OR $(95 \% \mathrm{Cl})^{\ddagger}$ & \multicolumn{2}{|l|}{$2.289(1.432-3.657)$} & \multicolumn{2}{|c|}{$2.492(1.067-5.829)$} & \multicolumn{2}{|c|}{$2.243(1.268-3.065)$} \\
\hline p-value & \multicolumn{2}{|l|}{0.001} & \multicolumn{2}{|c|}{0.035} & \multicolumn{2}{|c|}{0.005} \\
\hline \multicolumn{7}{|l|}{ CETP rs708272 (G>A) } \\
\hline GG, $n=34(M) ; 67(W)$ & $49(48.5)$ & $52(51.5)$ & $15(44.1)$ & $19(55.9)$ & $34(50.7)$ & $33(49.3)$ \\
\hline $\mathrm{GA}, \mathrm{n}=49(\mathrm{M}) ; 104(\mathrm{~W})$ & $74(48.4)$ & $79(51.6)$ & $19(38.8)$ & $30(6 \mid .2)$ & $55(52.9)$ & $49(47.1)$ \\
\hline$A A, n=12(M) ; 33(W)$ & $38(84.4)$ & $7(15.6)$ & $9(75.0)$ & $3(25.0)$ & $29(87.9)$ & $4(12.1)$ \\
\hline $\begin{array}{l}\text { OR }(95 \% \mathrm{Cl})^{\S} \\
\text { p-value }\end{array}$ & $\begin{array}{l}5.782(2.489-13.431) \\
<0.001\end{array}$ & & $\begin{array}{l}4.324(1.090-17 \\
0.037\end{array}$ & 50) & $\begin{array}{l}6.680(2.251-19 \\
0.001\end{array}$ & 20) \\
\hline CETP rs I 2 I $49545(\mathrm{G}>\mathrm{A})$ & & & & & & \\
\hline $\mathrm{GG}, \mathrm{n}=61(\mathrm{M}) ; 132(\mathrm{~W})$ & $101(52.3)$ & $92(47.7)$ & $25(41.0)$ & $36(59.0)$ & $76(57.6)$ & $56(42.4)$ \\
\hline $\mathrm{GA}, \mathrm{n}=3 \mathrm{I}(\mathrm{M}) ; 66(\mathrm{~W})$ & $53(54.6)$ & $44(45.4)$ & $16(51.6)$ & $15(48.4)$ & $37(56.1)$ & $29(43.9)$ \\
\hline $\mathrm{AA}, \mathrm{n}=3(\mathrm{M}) ; 6(\mathrm{~W})$ & 7 (77.8) & $2(22.2)$ & $2(66.7)$ & I (33.3) & $5(83.3)$ & I (16.7) \\
\hline OR $(95 \% \mathrm{Cl}){ }^{\ddagger}$ & $1.188(0.738-1.914)$ & & $1.620(0.696-3.7$ & & $1.032(0.576-1.8$ & \\
\hline$p$-value & 0.479 & & 0.263 & & 0.917 & \\
\hline
\end{tabular}

(Continued) 
Table 4 (Continued).

\begin{tabular}{|c|c|c|c|c|c|c|}
\hline \multirow[b]{2}{*}{ VLDL-cholesterol } & \multicolumn{2}{|l|}{ All, n (\%) } & \multicolumn{2}{|l|}{ Men, n (\%) } & \multicolumn{2}{|l|}{ Women, n (\%) } \\
\hline & $\begin{array}{l}\leq 0.75 \mathrm{mmol} / \mathrm{L} \\
(\mathrm{n}=149)\end{array}$ & $\begin{array}{l}>0.75 \mathrm{mmol} / \mathrm{L} \\
(\mathrm{n}=150)\end{array}$ & $\begin{array}{l}\leq 0.75 \mathrm{mmol} / \mathrm{L} \\
(\mathrm{n}=37)\end{array}$ & $\begin{array}{l}>0.75 \mathrm{mmol} / \mathrm{L} \\
(\mathrm{n}=58)\end{array}$ & $\begin{array}{l}\leq 0.75 \mathrm{mmol} / \mathrm{L} \\
(\mathrm{n}=\mid I 2)\end{array}$ & $\begin{array}{l}>0.75 \mathrm{mmol} / \mathrm{L} \\
(\mathrm{n}=92)\end{array}$ \\
\hline \multicolumn{7}{|l|}{ CETP rs376426I (C>A) } \\
\hline$C C, n=40(M) ; 86(W)$ & $42(33.3)$ & $84(66.7)$ & $9(22.5)$ & $31(77.5)$ & $33(38.4)$ & $53(61.6)$ \\
\hline $\mathrm{CA}, \mathrm{n}=43(\mathrm{M}) ; 97(\mathrm{~W})$ & $85(60.7)$ & $55(39.3)$ & $23(53.5)$ & $20(46.5)$ & $62(63.9)$ & $35(36.1)$ \\
\hline $\mathrm{AA}, \mathrm{n}=12(\mathrm{M}) ; 21(\mathrm{~W})$ & $22(66.7)$ & II (33.3) & $5(41.7)$ & $7(58.3)$ & $17(81.0)$ & $4(19.0)$ \\
\hline OR $(95 \% \mathrm{Cl})^{\ddagger}$ & \multicolumn{2}{|c|}{$3.242(2.004-5.245)$} & \multicolumn{2}{|c|}{$3.572(1.436-8.884)$} & \multirow{2}{*}{\multicolumn{2}{|c|}{$\begin{array}{l}3.253(1.822-5.809) \\
<0.001\end{array}$}} \\
\hline p-value & $<0.001$ & & 0.006 & & & \\
\hline \multicolumn{7}{|l|}{ CETP rs708272 (G>A) } \\
\hline $\mathrm{GG}, \mathrm{n}=34(\mathrm{M}) ; 67(\mathrm{~W})$ & $38(37.6)$ & $63(62.4)$ & $10(29.4)$ & $24(70.6)$ & $28(41.8)$ & $39(58.2)$ \\
\hline $\mathrm{GA}, \mathrm{n}=49(\mathrm{M}) ; 104(\mathrm{~W})$ & $71(46.4)$ & $82(53.6)$ & $18(36.7)$ & $31(63.3)$ & $53(51.0)$ & $51(49.0)$ \\
\hline$A A, n=12(M) ; 33(W)$ & $40(88.9)$ & $5(11.1)$ & $9(75.0)$ & $3(25.0)$ & 31 (93.9) & $2(6.1)$ \\
\hline OR $(95 \% \mathrm{Cl})^{\S}$ & \multicolumn{2}{|c|}{$10.642(4.065-27.859)$} & \multicolumn{2}{|c|}{$5.893(1.477-23.508)$} & \multirow{2}{*}{\multicolumn{2}{|c|}{$\begin{array}{l}\mid 7.222(3.995-74.238) \\
<0.00 \mid\end{array}$}} \\
\hline$p$-value & \multicolumn{2}{|c|}{$<0.001$} & \multicolumn{2}{|c|}{0.012} & & \\
\hline \multicolumn{7}{|l|}{ CETP rs I2I 49545 (G>A) } \\
\hline $\mathrm{GG}, \mathrm{n}=61(\mathrm{M}) ; 132(\mathrm{~W})$ & $87(45.1)$ & $106(54.9)$ & $19(31.1)$ & $42(68.9)$ & $68(51.5)$ & $64(48.5)$ \\
\hline $\mathrm{GA}, \mathrm{n}=31(\mathrm{M}) ; 66(\mathrm{~W})$ & $56(57.7)$ & $4 \mid(42.3)$ & $17(54.8)$ & $14(45.2)$ & $39(59.1)$ & $27(40.9)$ \\
\hline $\mathrm{AA}, \mathrm{n}=3(\mathrm{M}) ; 6(\mathrm{~W})$ & $6(66.7)$ & $3(33.3)$ & I (33.3) & $2(66.7)$ & $5(83.3)$ & I (I6.7) \\
\hline OR $(95 \% \mathrm{Cl})^{\ddagger}$ & \multicolumn{2}{|c|}{$1.717(1.063-2.772)$} & \multicolumn{2}{|c|}{$2.487(1.048-5.903)$} & \multirow{2}{*}{\multicolumn{2}{|c|}{$\begin{array}{l}\text { I.479 (0.825-2.652) } \\
0.189\end{array}$}} \\
\hline p-value & 0.026 & & 0.039 & & & \\
\hline LDL subclass & $\begin{array}{l}\text { Pattern A } \\
(n=188)\end{array}$ & $\begin{array}{l}\text { Pattern B } \\
(n=I I I)\end{array}$ & $\begin{array}{l}\text { Pattern A } \\
(n=47)\end{array}$ & $\begin{array}{l}\text { Pattern B } \\
(n=48)\end{array}$ & $\begin{array}{l}\text { Pattern A } \\
(n=14 I)\end{array}$ & $\begin{array}{l}\text { Pattern B } \\
(n=63)\end{array}$ \\
\hline \multicolumn{7}{|l|}{ CETP rs376426I (C>A) } \\
\hline$C C, n=40(M) ; 86(W)$ & $64(50.8)$ & $62(49.2)$ & $15(37.5)$ & $25(62.5)$ & $49(57.0)$ & $37(43.0)$ \\
\hline$C A, n=43(M) ; 97(W)$ & $96(68.6)$ & $44(3 \mid .4)$ & $24(55.8)$ & $19(44.2)$ & $72(74.2)$ & $25(25.8)$ \\
\hline $\mathrm{AA}, \mathrm{n}=12(\mathrm{M}) ; 21(\mathrm{~W})$ & $28(84.8)$ & $5(15.2)$ & $8(66.7)$ & $4(33.3)$ & $20(95.2)$ & I (4.8) \\
\hline OR $(95 \% \mathrm{Cl})^{\ddagger}$ & \multicolumn{2}{|c|}{$2.452(1.515-3.966)$} & \multicolumn{2}{|c|}{$2.319(1.006-5.343)$} & \multicolumn{2}{|c|}{$2.672(1.452-4.916)$} \\
\hline p-value & \multicolumn{2}{|c|}{$<0.001$} & \multicolumn{2}{|l|}{0.048} & \multicolumn{2}{|c|}{0.002} \\
\hline CETP rs708272 (G>A) & & & & & & \\
\hline $\mathrm{GG}, \mathrm{n}=34(\mathrm{M}) ; 67(\mathrm{~W})$ & $55(54.5)$ & $46(45.5)$ & $17(50.0)$ & $17(50.0)$ & $38(56.7)$ & $29(43.3)$ \\
\hline $\mathrm{GA}, \mathrm{n}=49(\mathrm{M}) ; 104(\mathrm{~W})$ & $94(6 \mathrm{I} .4)$ & $59(38.6)$ & $20(40.8)$ & $29(59.2)$ & $74(7 \mid .2)$ & $30(28.8)$ \\
\hline$A A, n=12(M) ; 33(W)$ & $39(86.7)$ & $6(13.3)$ & $10(83.3)$ & $2(16.7)$ & $29(87.9)$ & $4(12.1)$ \\
\hline OR $(95 \% \mathrm{Cl})^{\S}$ & $4.581(1.872-1)$ & & $6.216(1.282-30$ & & $3.819(1.282-11$. & 8I) \\
\hline$p$-value & $<0.001$ & & 0.023 & & 0.016 & \\
\hline CETP rsI2I49545 (G>A) & & & & & & \\
\hline$G G, n=61(M) ; 132(W)$ & II $(59.6)$ & $78(40.4)$ & $27(44.3)$ & $34(55.7)$ & $88(66.7)$ & $44(33.3)$ \\
\hline $\mathrm{GA}, \mathrm{n}=31(\mathrm{M}) ; 66(\mathrm{~W})$ & $66(68.0)$ & $31(32.0)$ & $18(58.1)$ & $13(4 \mid .9)$ & $48(72.7)$ & $18(27.3)$ \\
\hline $\mathrm{AA}, \mathrm{n}=3(\mathrm{M}) ; 6(\mathrm{~W})$ & $7(77.8)$ & $2(22.2)$ & $2(66.7)$ & I (33.0) & $5(83.3)$ & I (I6.7) \\
\hline OR $(95 \% \mathrm{Cl})^{\ddagger}$ & $1.500(0.909-2.4$ & & $1.799(0.769-4.2$ & & $1.395(0.738-2.6$ & \\
\hline$p$-value & 0.113 & & 0.176 & & 0.306 & \\
\hline
\end{tabular}

Notes: ${ }^{\dagger}$ Classified as HDL-cholesterol $\geq 1.04 \mathrm{mmol} / \mathrm{L}$ and $<1.04 \mathrm{mmol} / \mathrm{L}$ for men and $\geq 1.30 \mathrm{mmol} / \mathrm{L}$ and $<1.30 \mathrm{mmol} / \mathrm{L}$ for women; ${ }^{38}$ triglycerides $<1.70 \mathrm{mmol} / \mathrm{L}$ and $\geq 1.70 \mathrm{mmol} /$ $\mathrm{L}^{38} \mathrm{VLDL}$-cholesterol $<0.75 \mathrm{mmol} / \mathrm{L}$ and $\geq 0.75 \mathrm{mmol} / \mathrm{L}$ (median concentration); LDL subclass Pattern A: mean LDL particle size $>26.50 \mathrm{~nm}$; Pattern B: mean LDL particle size $\leq 26.50 \mathrm{~nm}^{36}{ }^{\ddagger}$ Odds ratio (95\% confidence interval): Recessive genetic model for CETP rs376426I: CC vs AA+CA and CETP rs 12।49545: GG vs AA+GA. ${ }^{8}$ Odds ratio (95\% confidence interval): Dominant genetic model for CETP rs708272: GG+GA vs AA. "The odds ratio and the $p$-value are not calculated because the number of hypo HDLcholesterolemia in AA genotype group is zero. 
Table 5 Logistic Regression Analysis Between CETP Genetic Variants, Potential Confounders and Atherogenic Dyslipidemia Complex ${ }^{\dagger}$

\begin{tabular}{|c|c|c|c|c|c|c|c|}
\hline \multirow[t]{3}{*}{ Dependent Variable } & \multirow[t]{3}{*}{ Independent Variables } & \multicolumn{6}{|c|}{ Logistic Regression $\ddagger$} \\
\hline & & \multicolumn{2}{|l|}{ All } & \multicolumn{2}{|l|}{ Men } & \multicolumn{2}{|l|}{ Women } \\
\hline & & OR $(95 \% \mathrm{Cl})$ & p-value & OR (95\% Cl) & p-value & OR (95\% Cl) & $p$-value \\
\hline \multirow[t]{4}{*}{ Model $\left.\right|^{\S}$} & rs376426I & $2.11(1.27-3.50)$ & 0.004 & $2.23(0.97-5.14)$ & 0.061 & $2.54(1.26-5.11)$ & 0.009 \\
\hline & rs708272 & $2.12(1.29-3.49)$ & 0.003 & $1.64(0.68-3.92)$ & 0.271 & $2.37(1.24-4.53)$ & 0.009 \\
\hline & HOMA-IR & $1.47(1.27-1.7 I)$ & $<0.001$ & $1.32(1.06-1.64)$ & 0.013 & $1.57(1.27-1.92)$ & $<0.001$ \\
\hline & Constant & 0.003 & $<0.001$ & 0.019 & 0.001 & 0.000 & $<0.001$ \\
\hline \multirow[t]{3}{*}{ Model $2^{\pi}$} & rs376426I and rs708272 & $2.99(1.78-5.02)$ & $<0.001$ & $2.90(1.16-7.25)$ & 0.023 & $3.40(I .72-6.7 I)$ & $<0.001$ \\
\hline & HOMA-IR & $1.45(1.25-1.67)$ & $<0.001$ & $1.31(1.06-1.62)$ & 0.014 & $1.55(1.26-1.90)$ & $<0.001$ \\
\hline & Constant & 0.008 & $<0.001$ & 0.010 & $<0.001$ & 0.001 & 0.001 \\
\hline
\end{tabular}

Notes: ${ }^{\dagger}$ Atherogenic dyslipidemia complex; all features of reduced HDL-C, elevated triglycerides and LDL subclass pattern B. ${ }^{\ddagger}$ Data are from logistic regression with backward Wald method, adjusted age, BMI, systolic and diastolic blood pressure, HOMA-IR and statin dosage; OR (95\% Cl): odds ratio (95\% confidential interval). ${ }^{\S}$ Variables entered in method: rs376426I, rs708272, and rs /2149545. "Variables entered in method: combination of variants at rs376426I and rs708272. The detail of the combination groups is shown in supplementary Table I.

genotypes) exhibited a stronger association with atherogenic dyslipidemia complex overall $(\mathrm{OR}=2.99,95 \% \mathrm{CI}$ : 1.78-5.02) and in both sexes (OR, 2.90; 95\% CI, 1.16-7.25 for men and OR, 3.40; 95\% CI, 1.72-6.71 for women).

\section{Discussion}

In this study, we demonstrated the relationship of residual dyslipidemia as a function of CETP variants in patients treated with statins. Our results indicated that CETP polymorphisms of rs3764261 and rs708272, but not rs12149545, were significantly associated with the risk of atherogenic dyslipidemia. Specific markers associated with CETP polymorphisms were the concentrations of HDL-C, apoA-I, triglycerides, VLDL-C, and large LDL (LDL1-C) and mean LDL particle size. Our results agree with those of population-based studies but differ from others in that all our patients were being treated with statins. ${ }^{21-23,27}$ However, no significant differences were observed in TC, LDL-C, and apoB levels when stratified by CETP variants. The measurement of apo B levels is known to be highly correlated with the total number of atherogenic particles. Thus, it was implied that CETP variants may not contribute to the statin action to increase LDL receptor activity in our patients.

Chapman et al ${ }^{19}$ indicated that statins not only reduced the elevated levels of atherogenic apoB-containing lipoproteins by lipolysis and hepatic removal by increasing LDL receptor activity but also attenuated CETP activity after decreased CETP expression. Although statin treatment reduces CETP activity by up to $30 \%$, considerable interindividual variation is observed ${ }^{39,40}$ partly due to genetic variants within CETP. ${ }^{41}$ A large-scale metaanalysis of genome-wide association studies revealed that the minor allele of CETP rs3764261 (A allele) was strongly associated with lower CETP mRNA expression levels in the hepatic tissue and with a higher HDL-C level in response to statin treatment. ${ }^{42}$ Consistent with this report, we found that carriers of the $\mathrm{CC}$ genotype were more likely to demonstrate elevated atherogenic risk markers relative to those with the allele A (AA and CA genotypes) in our cohort of statin-treated patients. Many studies have indicated the role of CETP rs708272 polymorphism in reducing CETP activities and HDL-C levels. ${ }^{18,43-46}$ Our results in a Thai population revealed that the $\mathrm{G}$ allele (combined GG and GA genotypes) was associated with a more favorable atherogenic dyslipidemia risk profile than the minor AA genotype. Thus, because of a strong association with atherogenic biomarkers, CETP polymorphisms may affect the effectiveness in patients receiving statins.

Carriers of the common genotypes of CETP rs3764261 (CC genotype) or rs708272 (GG and GA genotypes) demonstrated an increased risk of atherogenic dyslipidemia. Notably, the relationships were sex-specific: the risk was significantly associated with the CETP rs3764261 and the CETP rs708272 polymorphisms only in women. Results on sex specificity in the association between CETP rs708272 polymorphism and serum lipid levels have been inconsistent. ${ }^{22,28,47-50}$ In a Jewish population, CETP rs 708272 polymorphism exerted a specific effect on lipid in women but not men. ${ }^{47}$ Moreover, the associations 
were noted in black South African women, ${ }^{48}$ obese women, ${ }^{49}$ and Indian Sikh women. ${ }^{22}$ By contrast, no significant associations were observed in Han Chinese women. ${ }^{28}$ Another study presented the association with dyslipidemia and the risk of myocardial infarction among white men in Western Siberia. ${ }^{50}$ Although the underlying mechanisms that explain sex-dependent CETP splicing effects on ASCVD risk are unclear, sex-related differences may reflect dissimilarities in the regulatory function of CETP in lipid exchange.

In our population, individuals with dyslipidemia more often carried the common alleles of CETP rs3764261 and rs708272, whereas those without atherogenic dyslipidemia more often carried the minor alleles. Notably, gene-gene interaction analysis revealed that the combination of CETP rs3764261 with rs708272 variants was associated with atherogenic dyslipidemia, regardless of sex (Table 5). Moreover, both variants exhibited the complementary risk of showing a stronger association with atherogenic dyslipidemia features than single variants rs3764261 and rs708272. Consistent with our findings, a study on Indian Sikh patients with diabetes revealed a strong interaction of rs3764261 and rs708272 in the association between CETP activity and HDL-C levels. ${ }^{22}$ Another study using the Japan Multi-Institutional Collaborative Cohort revealed that the CETP rs3764261 polymorphism was associated with high HDL-C levels with any combination of genes in the reverse cholesterol transport system (ie, $A B C A 1$, $A P O A 1$, and $S R-B 1$ polymorphisms). ${ }^{51}$ Taken together, the findings suggest that the combination of CETP rs3764261 and rs708272 is a promising genetic marker for atherogenic dyslipidemia risk estimation in statintreated patients.

Among CETP polymorphisms, rs3764261, located in the promoter region, and rs708272, located in the first intron region, have been widely studied for their roles in altered lipid metabolism. ${ }^{18,20-29,41-50}$ Little is known regarding the relationship between rs12149545 and dyslipidemia, especially in statin-treated patients. Our results demonstrated that rs12149545, located in the promoter region similar to rs3764261, was not significantly associated with any feature of dyslipidemia in statin-treated Thai patients. This CETP variant may not likely affect CETP production or its activity.

CETP variants have received recognition as a potential contributor to ASCVD risk, but it is unclear if this relationship differs based on statin treatment. ${ }^{18,43,52}$ Carlquist et al ${ }^{43}$ demonstrated that the
CETP rs708272 polymorphism was associated with the incidence of clinical events (death or myocardial infarction) in statin-treated patients but not in patients not taking statins. By contrast, in Mendelian randomization analyses involving participants from 14 large studies, the CETP genetic score created by combined, multiple, independently inherited CETP variants related to the targets of CETP inhibitor (ie, rs3764261, rs1800775, rs1864163, rs9929488, rs9989419, rs12708967, rs289714, and rs5880) were associated with a lower risk of ASCVD events in patients not taking statin but not in patients taking statin. ${ }^{18}$ Moreover, major trials evaluating the effect of CETP inhibitors on reducing ASCVD events have yielded inconclusive results: some demonstrating harm, some demonstrating no benefit, and some reporting efficacy. ${ }^{53-56}$ These inconsistent results suggest that a multifactorial network of pathways influences metabolic outcomes in patients treated with CETP inhibitors. According to our findings in a Thai cohort, CETP polymorphisms potentially affect HDL, VLDL, and LDL metabolism, specifically in the context of the atherogenic dyslipidemia complex. Increased understanding of the contributions of the residual risk factors in different patient populations may facilitate more effective precision medicine approaches to prevent ASCVD events. Genetic testing of CETP may be essential for early prediction of residual ASCVD risk, especially in patients with high-risk factors such as type 2 diabetes or metabolic syndrome. Due to the relation between the CETP rs3764261 and rs708272 variants and the atherogenic dyslipidemia complex in statintreated patients, additional treatment of CETP inhibitors in patients with metabolic abnormalities may decrease the residual ASCVD risk.

A limitation of this study is its retrospective crosssectional design; a prospective study is warranted to verify the association. Additionally, whether the CETP rs3764261 and rs708272 polymorphisms contribute to ASCVD risks needs to be confirmed with further prospective studies. Second, all patients in the present study were ethnically Thai. The expression of CETP variants may vary across ethnic groups. Future studies should include other ethnicities. Third, female patients were predominant in our study ( $68.2 \%$ women, $31.8 \%$ men), which probably reflected the higher ASCVD risk in women than in men. Fourth, although we adjusted for age, BMI, blood pressure, insulin resistance, and statin dosage, which are known risk factors for atherogenic 
dyslipidemia, others such as the duration and type of previous medication, dietary intake, physical activity, and family history were not accounted for. Finally, we assumed that all statins were equally effective.

In conclusion, in adult statin-treated Thai patients, polymorphisms of CETP rs3764261 (C>A) and rs708272 $(\mathrm{G}>\mathrm{A})$ were associated with dyslipidemia. A higher risk of atherogenic dyslipidemia was observed in patients with CETP variants, rs3764261 CC or rs $708272 \mathrm{GG}$ and GA. However, the significant associations of CETP rs3764261 or rs708272 polymorphisms with atherogenic dyslipidemia, independent of HOMA-IR, were sex specific. The combination of these CETP variants can improve the atherogenic dyslipidemia risk estimation, regardless of sex. CETP genotyping in statin-treated patients may help predict the alterations in HDL, VLDL, and LDL metabolisms. Accumulating evidence has indicated that the effectiveness of statin therapy relies on personalized medicine for lipid management, particularly for patients with metabolic disorders. In this regard, genetic testing of CETP may allow preventative measures that may help individualize treatment for reducing the residual ASCVD risk in statin-treated patients.

\section{Ethics Approval and Consent to Participate}

The study protocol was reviewed and approved by the ethics committee of Ramathibodi Hospital (Approval reference number ID MURA2019/984). All methods were carried out in accordance with the ethical guidelines of the 1975 Declaration of Helsinki.

\section{Acknowledgments}

The authors acknowledge to all patients who participated in this study. We thank Natchaya Vanwong, Jirapa Kerdmongkol and all staffs in Divisions of Clinical Chemistry and Pharmacogenomics and Personalized Medicine, Department of Pathology, Faculty of Medicine, Ramathibodi Hospital, Mahidol University, for data collection and management.

\section{Funding}

This research project is supported by grant of the Faculty of Medicine, Ramathibodi Hospital, Mahidol University [grant number RF_63025].

\section{Disclosure}

The authors have no conflicts of interest to declare.

\section{References}

1. Arnett DK, Blumenthal RS, Albert MA, et al. ACC/AHA guideline on the primary prevention of cardiovascular disease a report of the American College of Cardiology/American Heart Association task force on clinical practice guidelines. Circulation. 2019;2019(140): e596-e646.

2. Stancu C, Sima A. Statins: mechanism of action and effects. $J$ Cell Mol Med. 2001;5:378-387. doi:10.1111/j.1582-4934.2001.tb00172.x

3. Ference BA, Yoo W, Alesh I. Effect of long-term exposure to lower low-density lipoprotein cholesterol beginning early in life on the risk of coronary heart disease: a mendelian randomization analysis. $J \mathrm{Am}$ Coll Cardiol. 2012;60:2631-2639. doi:10.1016/j.jacc.2012.09.017

4. Baigent C, Keech A, Kearney PM, et al. Cholesterol Treatment Trialists' (CTT) collaborators: efficacy and safety of cholesterol-lowering treatment: prospective meta-analysis of data from 90056 participants in 14 randomised trials of statins. Lancet. 2005;336:1267-1278.

5. Mihaylova B, Emberson J, Blackwell L, et al.; Cholesterol Treatment Trialists' (CTT) Collaborators. The effects of lowering LDL cholesterol with statin therapy in people at low risk of vascular disease: meta-analysis of individual data from 27 randomised trials. Lancet. 2012;380:581-590.

6. Ridker PM, Pradhan A, MacFadyen JG, et al. Cardiovascular benefits and diabetes risks of statin therapy in primary prevention: an analysis from the JUPITER trial. Lancet. 2012;380:565-571. doi:10.1016/ S0140-6736(12)61190-8

7. Davies JT, Delfino SF, Feinberg CE, et al. Current and emerging uses of statins in clinical therapeutics: a review. Lipid Insights. 2016;9:13-29. doi:10.4137/LPI.S37450

8. Akyea RK, Kai J, Qureshi N, et al. Sub-optimal cholesterol response to initiation of statins and future risk of cardiovascular disease. Heart. 2019;105:975-981. doi:10.1136/heartjnl-2018-314253

9. Rana JS, Liu JY, Moffet HH, et al. Metabolic dyslipidemia and risk of coronary heart disease in 28,318 adults with diabetes mellitus and low-density lipoprotein cholesterol $<100 \mathrm{mg} / \mathrm{dl}$. Am J Cardiol. 2015;116:1700-1704.

10. Nichols GA, Philip S, Reynolds K, et al. Increased cardiovascular risk in Hypertriglyceridemic patients with statin-controlled LDL cholesterol. J Clin Endocrinol Metab. 2018;103:3019-3027. doi:10.1210/jc.2018-00470

11. Lawler PR, Akinkuolie AO, Chu AY, et al. Atherogenic lipoprotein determinants of cardiovascular disease and residual risk among individuals with low low-density lipoprotein cholesterol. $J$ Am Heart Assoc. 2017;6:e05549. doi:10.1161/JAHA.117.005549

12. Nichols GA, Philip S, Reynolds K, et al. Increased residual cardiovascular risk in patients with diabetes and high versus normal triglycerides despite statin-controlled LDL cholesterol. Diabetes Obes Metab. 2019;21:366-371.

13. Sarwar N, Danesh J, Eiriksdottir G, et al. Triglycerides and the risk of coronary heart disease: 10,158 incident cases among 262,525 participants in 29 Western prospective studies. Circulation. 2007;115:450-458. doi:10.1161/CIRCULATIONAHA.106.637793

14. Barter PJ, Brewer HB Jr, Chapman MJ, et al. Cholesteryl ester transfer protein: a novel target for raising HDL and inhibiting atherosclerosis. Arterioscler Thromb Vasc Biol. 2003;23:160-167. doi:10.1161/01.ATV.0000054658.91146.64

15. Chapman MJ, Goff WL, Guerin M, et al. Cholesteryl ester transfer protein: at the heart of the action of lipid-modulating therapy with statins, fibrates, niacin, and cholesteryl ester transfer protein inhibitors. Eur Heart J. 2010;31:149-164.

16. Guerin M, Lassel TS, Goff WL, et al. Action of atorvastatin in combined hyperlipidemia: preferential reduction of cholesteryl ester transfer from HDL to VLDL1 particles. Arterioscler Thromb Vasc Biol. 2000;20:189-197. doi:10.1161/01.ATV.20.1.189 
17. Karalis IK, Bergheanu SC, Wolterbeek R, et al. Effect of increasing doses of rosuvastatin and atorvastatin on apolipoproteins, enzymes and lipid transfer proteins involved in lipoprotein metabolism and inflammatory parameters. Curr Med Res Opin. 2010;26:2301-2313. doi:10.1185/03007995.2010.509264

18. Ference BA, Kastelein JJP, Ginsberg HN, et al. Association of genetic variants related to CETP inhibitors and statins with lipoprotein levels and cardiovascular risk. JAMA. 2017;318:947-956. doi:10.1001/jama.2017.11467

19. Chapman MJ, Orsoni A, Robillard P, et al. Duality of statin action on lipoprotein subpopulations in the mixed dyslipidemia of metabolic syndrome: quantity vs quality over time and implication of CETP. J Clin Lipidol. 2018;12:784-800.

20. Blauw LL, Li-Gao R, Noordam R, et al. CETP (cholesteryl ester transfer protein) concentration: a genome-wide association study followed by Mendelian randomization on coronary artery disease. Circ Genom Precis Med. 2008;11:e002034.

21. Thompson A, Di Angelantonio E, Sarwar N, et al. Association of cholesteryl ester transfer protein genotypes with CETP mass and activity, lipid levels, and coronary risk. JAMA. 2008;299:2777-2788. doi:10.1001/jama.299.23.2777

22. Schierer A, Been LF, Ralhan S, et al. Genetic variation in cholesterol ester transfer protein, serum CETP activity, and coronary artery disease risk in Asian Indian diabetic cohort. Pharmacogenet Genomics. 2012;22:95-104. doi:10.1097/FPC.0b013e32834dc9ef

23. Ordovas JM, Cupples LA, Corella D, et al. Association of cholesteryl ester transfer protein-TaqIB polymorphism with variations in lipoprotein subclasses and coronary heart disease risk: the Framingham study. Arterioscler Thromb Vasc Biol. 2000;20:1323-1329. doi:10.1161/01.ATV.20.5.1323

24. Su Y, Wu K, Zhang Y, et al. Association between CETP I405 $\mathrm{V}$ polymorphism and hypertension in Hainan Han population. J Chin Gerontol. 2016;36:4458-4459.

25. Schechter CB, Barzilai N, Crandall JP, et al. Cholesteryl ester transfer protein (CETP) genotype and reduced CETP levels associated with decreased prevalence of hypertension. Mayo Clin Proc. 2010;85:522. doi: $10.4065 / \mathrm{mcp} .2009 .0594$

26. Li H, Xiao H, Zhang Y, et al. Relationship between CETP gene polymorphism and type 2 diabetes in Uygur. Chin Public Health. 2009;25:1430-1432.

27. Inazu A, Brown ML, Hesler CB, et al. Increased high-density lipoprotein levels caused by a common cholesteryl-ester transfer protein gene mutation. N Engl J Med. 1990;323:1234-1238. doi:10.1056/ NEJM199011013231803

28. Cai G, Shi G, Huang Z. Gender specific effect of CETP rs 708272 polymorphism on lipid and atherogenic index of plasma levels but not on the risk of coronary artery disease: a case-control study. Medicine. 2018;49:e13514. doi:10.1097/MD.0000000000013514

29. McCaffery JM, Ordovas JM, Huggins GS, et al. Weight gain prevention buffers the impact of CETP rs3764261 on high density lipoprotein cholesterol in young adulthood: the Study of Novel Approaches to Weight Gain Prevention (SNAP). Nutr Metab Cardiovasc Dis. 2018;28:816-821. doi:10.1016/j.numecd.2018.02.018

30. Guo S, Hu Y, Ding Y, et al. Association between eight functional polymorphisms and haplotypes in the Cholesterol Ester Transfer Protein (CETP) gene and dyslipidemia in national minority adults in the Far West Region of China. Int J Environ Res Public Health. 2015;12:15979-15992. doi:10.3390/ijerph121215036

31. Hou H, Ma R, Guo H, et al. Association between six CETP polymorphisms and metabolic syndrome in Uyghur adults from Xinjiang, China. Int J Environ Res Public Health. 2017;14:653.

32. Kooner JS, Chambers JC, Aguilar-Salinas CA, et al. Genome-wide scan identifies variation in MLXIPL associated with plasma triglycerides. Nat Genet. 2008;40:149-151. doi:10.1038/ng.2007.61
33. Lu Y, Tayebi N, Li H, et al. Association of CETP Taq1B and '629C > A polymorphisms with coronary artery disease and lipid levels in the multi-ethnic Singaporean population. Lipids Health Dis. 2013;12:1-13. doi:10.1186/1476-511X-12-85

34. Wang X, Magkos F, Mittendorfer B. Sex differences in lipid and lipoprotein metabolism: it's not just about sex hormones. $J$ Clin Endocrinol Metab. 2011;96:885-893.

35. Puangpetch A, Srisawasdi P, Unaharassamee W, et al. Association between polymorphisms of LEP, LEPR, DRD2, HTR2A and HTR2C genes and risperidone- or clozapine-induced hyperglycemia. Pharmgenomics Pers Med. 2019;12:155-166.

36. Hoefner DM, Hodel SD, O'Brien JF, et al. Development of a rapid, quantitative method for LDL subfractionation with use of the Quantimetrix Lipoprint LDL System. Clin Chem. 2001;47:266-274. doi:10.1093/clinchem/47.2.266

37. Matthews DR, Hosker JP, Rudenski AS, et al. Homeostasis model assessment: insulin resistance and beta-cell function from fasting plasma glucose and insulin concentrations in man. Diabetologia. 1985;28:412-419. doi:10.1007/BF00280883

38. National Cholesterol Education Program (NCEP). Expert panel on detection, evaluation, and treatment of high blood cholesterol in adults (Adult Treatment Panel III). Third Report of the National Cholesterol Education Program (NCEP) expert panel on detection, evaluation, and treatment of high blood cholesterol in adults (Adult Treatment Panel III) final report. Circulation. 2002;106:3143-3421.

39. Ahnadi CE, Berthezene F, Ponsin G. Simvastatin-induced decrease in the transfer of cholesterol esters from high density lipoproteins to very low and low density lipoproteins in normolipidemic subjects. Atherosclerosis. 1993;99:219-228. doi:10.1016/0021-9150(93)90024-O

40. Guerin M, Egger P, Soudant C, et al. Dose-dependent action of atorvastatin in type IIB hyperlipidemia: preferential and progressive reduction of atherogenic apoB-containing lipoprotein subclasses (VLDL-2, IDL, small dense LDL) and stimulation of cellular cholesterol efflux. Atherosclerosis. 2002;163:287-296. doi:10.1016/S00219150(02)00037-0

41. Kuivenhoven JJW, Zwinderman AH, et al. The role of a commJAon variant of the cholesteryl ester transfer protein gene in the progression of coronary atherosclerosis. The Regression Growth Evaluation Statin Study Group. N Engl J Med. 1998;338:86-93. doi:10.1056/ NEJM199801083380203

42. Postmus I, Warren HR, Trompet S, et al. Meta-analysis of genome-wide association studies of HDL cholesterol response to statins. J Med Genet. 2016;53:835-845. doi:10.1136/jmedgenet-2016-103966

43. Carlquist JF, Muhlestein JB, Horne BD, et al. The cholesteryl ester transfer protein Taq1B gene polymorphism predicts clinical benefit of statin therapy in patients with significant coronary artery disease. $\mathrm{Am}$ Heart J. 2003;146:1007-1014. doi:10.1016/S0002-8703(03)00501-5

44. Wang J, Wang LJ, Zhong Y, et al. CETP gene polymorphisms and risk of coronary atherosclerosis in a Chinese population. Lipids Health. 2013;12;176.

45. Whiting BM, Anderson JL, Muhlestein JB, et al. Candidate gene susceptibility variants predict intermediate end points but not angiographic coronary artery disease. Am Heart J. 2005;150:243-250. doi:10.1016/j.ahj.2004.08.034

46. Niu W, Qi Y. Circulating cholesteryl ester transfer protein and coronary heart disease: mendelian randomization meta-analysis. Circ Cardiovasc Genet. 2015;8:114-121. doi:10.1161/CIRCGENE TICS.114.000748

47. Kark JD, Sinnreich R, Leitersdorf E, et al. Taq1B CETP polymorphism, plasma CETP, lipoproteins, apolipoproteins and sex differences in a Jewish population sample characterized by low HDL-cholesterol. Atherosclerosis. 2000;151:509-518. doi:10.1016/S0021-9150(99) 00408-6 
48. Ellman N, Keswell D, Collins M, et al. Ethnic differences in the association between lipid metabolism genes and lipid levels in black and white South African women. Atherosclerosis. 2015;240:311-317. doi:10.1016/j.atherosclerosis.2015.03.027

49. Heilbronn LK, Noakes M, Clifton PM. Association between HDL cholesterol and the Taq1B polymorphism in the cholesterol ester transfer protein gene in obese women. Atherosclerosis. 2002;162:419-424. doi:10.1016/S0021-9150(01)00733-X

50. Semaev S, Shakhtshneider E, Orlov P, et al. Association of RS708272 (CETP Gene Variant) with lipid profile parameters and the risk of myocardial infarction in the white population of western Siberia. Biomolecules. 2019;9:739. doi:10.3390/biom9110739

51. Nakamura A, Niimura H, Kuwabara K, et al. Gene-gene combination effect and interactions among ABCA1, APOA1, SR-B1, and CETP polymorphisms for serum high-density lipoprotein-cholesterol in the Japanese population. PLoS One. 2013;8:e82046.

52. Millwood IY, Bennett DA, Holmes MV, et al. China Kadoorie Biobank Collaborative Group. Association of CETP gene variants with risk for vascular and nonvascular diseases among Chinese adults. JAMA Cardiol. 2018;3:34-43. doi:10.1001/jamacardio.201 7.4177
53. Barter PJ, Caulfield M, Eriksson M, et al. ILLUMINATE Investigators. Effects of torcetrapib in patients at high risk for coronary events. $N$ Engl J Med. 2007;357:2109-2122. doi:10.1056/ NEJMoa0706628

54. Schwartz GG, Olsson AG, Abt M, dal-OUTCOMES Investigators, et al. Effects of dalcetrapib in patients with a recent acute coronary syndrome. $N$ Engl J Med. 367;2012:2089-2099. doi:10.1056/ NEJMoa1206797

55. Lincoff AM, Nicholls SJ, Riesmeyer JS, ACCELERATE Investigators, et al. Evacetrapib and cardiovascular outcomes in high-risk vascular disease. $N$ Engl J Med. 376;2017:1933-1942. doi:10.1056/NEJMoa1609581

56. Bowman L, Hopewell JC, Chen F, et al.; HPS3/TIMI55-REVEAL Collaborative Group. Effects of anacetrapib in patients with atherosclerotic vascular disease. $N$ Engl J Med. 2017;377:1217-1227.

\section{Publish your work in this journal}

Pharmacogenomics and Personalized Medicine is an international, peer-reviewed, open access journal characterizing the influence of genotype on pharmacology leading to the development of personalized treatment programs and individualized drug selection for improved safety, efficacy and sustainability. This journal is indexed on the American Chemical Society's Chemical Abstracts Service (CAS). The manuscript management system is completely online and includes a very quick and fair peer-review system, which is all easy to use. Visit http://www.dovepress.com/testimonials.php to read real quotes from published authors. 